\title{
PLASMA CONCENTRATIONS \\ OF OESTRADIOL-17 $\beta$ AND SERUM LEVELS \\ OF FOLLICLE STIMULATING HORMONE IN THE IMMATURE FEMALE RAT
}

\author{
Henriette M. A. MEIJS-ROELOFS, J.-Th. J. UILENBROEK, \\ F. H. de JONG and R. W. WELSCHEN \\ Department of Anatomy, \\ Rotterdam Medical Faculty, The Netherlands
}

Plasma oestradiol- $\mathrm{I} 7 \beta\left(\mathrm{E}_{2}\right)$ was measured in peripheral plasma of immature female rats of the R-Amsterdam strain, a Wistar substrain, ranging from 5-35 days of age. Estimations of $\mathrm{E}_{2}$ were made by radio-immunoassay with the antibody described by EXLEY, JOHNSON and DEAN (I97I). Maximal plasma $\mathrm{E}_{2}$ levels of $55-60 \mathrm{pg} / \mathrm{ml}$ were found on days Io-I5; from days $25-35$, low to undetectable $\mathrm{E}_{2}$ levels were found. The pattern of $\mathrm{E}_{2}$ levels during the prepuberal period showed a close resemblance to that of follicle stimulating hormone (FSH), as measured earlier by RIA (Meijs-Roelofs, UilenBroek, Osman and Welschen, I973). The $\mathrm{E}_{2}$ measured appeared to be of ovarian origin : Ovariectomy (OVX) performed on day I3 resulted in a clearly decreased $\mathrm{E}_{\mathbf{2}}$ level two lays later ( $\mathrm{I} 3 \mathrm{pg} / \mathrm{ml}$ ), as compared to the littermate control value $(46 \mathrm{pg} / \mathrm{ml})$. Adrenalectomy did not change the level of circulating $\mathrm{E}_{\mathbf{2}}(54 \mathrm{pg} / \mathrm{ml})$.

A clear inhibitory feed-back action of the circulating oestradiol on the FSH secretion at this early age was demonstrated by the following findings : OVX on day I3 resulted in a signicantly increased serum FSH level two days later (I $770 \mathrm{ng}$ NIAMD-rat-FSH RP-I/ml) as compared to control values (I $033 \mathrm{ng} / \mathrm{ml}$ ). This increase could be prevented by daily s.c. injections of at least $0 . \mathrm{I} \mu \mathrm{g}$ oestradiol benzoate/roo $\mathrm{g}$ body weight, resulting in a peripheral plasma $\mathrm{E}_{2}$ concentration of $50 \mathrm{pg} / \mathrm{ml}$.

Our data seem to be rather paradoxical : an inhibitory feedback action is operative already at I3-I5 days of age, but $\mathrm{FSH}$ and $\mathrm{E}_{2}$ levels are both extremely high at that age. This discrepancy could be due to a low sensitivity of the inhibitory feedback system or, more likely, to the high concentration of specific $\mathrm{E}_{\mathbf{z}}$ binding proteins in plasma of immature rats (NunEz, ENGElmanN, BENASSAYAG, Savu, Crepy and Jayle, I97I ; Raynaud, Mercier-Bodard and Baulieu, I97I). 


\section{CONCENTRATIONS PLASMATIQUES DE L'OESTRADIOL I $7 \beta$ ET DE IA FSH CHEZ LA RATTE IMMATURE}

Chez des Rattes âgées de 5 à 35 jours, le niveau d'œstradiol plasmatique est à son maximum entre les jours Io et $I_{5}(55$ à $60 \mathrm{pg} / \mathrm{ml})$. A partir du jour 25 le taux d'œstradiol est très faible et le dosage n'est plus possible. La variation des concentrations d'œstradiol pendant la période prépubertaire est étroitement comparable à celle de la FSH plasmatique (MEIJS-RoErofs et al., I973).

Cet ostradiol est bien d'origine ovarienne, car la castration au jour 13 provoque une chute de l'œstradiol circulant (au jour I5 : I3 pg/ml chez les castrés, contre $46 \mathrm{pg} / \mathrm{ml}$ chez les témoins), alors que la surrénalectomie est sans effet $(54 \mathrm{pg} / \mathrm{ml})$.

La rétroaction négative exercée par l'œstradiol sur la sécrétion de FSH existe car l'ovariectomie au jour I3 provoque une élévation significative de la FSH circulante (au jour I5 : I $770 \mathrm{ng} / \mathrm{ml}$ chez les castrés contre I $033 \mathrm{ng} / \mathrm{ml}$ chez les témoins) et l'injection journalière d'une dose minimum de benzoate d'œstradiol de $0, \mathrm{I} \mu \mathrm{g} / \mathrm{xoo} \mathrm{g}$ prévient cette élévation de la FSH.

Ces résultats semblent paradoxaux. Une rétroaction négative des œstrogènes sur la FSH existe dès l'âge de I3-I5 jours, et à cet âge les taux d'œestradiol et de FSH circulants sont tous deux très élevés. La cause de cette contradiction pourrait être la faible sensibilité du système de rétroaction négative ou, plus probablement, la forte concentration du plasma de Ratte immature en protéines liant spécifiquement l'œstradiol.

\section{REFERENCES}

Exley D., Johnson M. W., Dean P. D. G., 197r. Antisera highly specific for I7 $\beta$-œstradiol. Steroids, 18, 605-620.

Meijs-Roelofs H. M. A., Uilenbroek J. Th. J., Osman P., Welschen R., I973. Serum levels of gonadotrophins and follicular growth in prepuberal rats. Int. Congr. Series Excerpta Medica, Copenhagen (in press).

Nunez M. E., Engelman F., Benassayag C., Savu L., Jayle M. M. F., 197r. Mise en évidence d'une fraction protéique liant les cestrogènes dans le sérum de rats impubères. C. R. Acad. Sci., Paris, Série D, 272, $2396-2399$.

Raynaud J.-P., Mercier-Bodard C., Baulieu E. E., I97I. Rat estradiol binding plasma protein (EBP). Steroids, 18, $767-784$. 